\title{
Hippo Signaling Pathway Reveals a Spatio- Temporal Correlation with the Size of Primordial Follicle Pool in Mice
}

\author{
Cheng Xianga,b Jia Lia Liaoliao Hu ${ }^{a}$ Jian Huang ${ }^{a}$ Tao Luo ${ }^{c}$ Zhisheng Zhong ${ }^{d}$ \\ Yuehui Zhenga Liping Zheng ${ }^{\text {a }}$ \\ aMedical Teaching Laboratory Center, Jiangxi Medical College, Nanchang University, Nanchang, \\ 'Department of Gynaecology and Obstetrics, People's Hospital of Huangmei County, Huanggang City, \\ Hubei, 'Life Sciences Institute, Nanchang University, Nanchang, 'Reproductive Medical Center, The \\ Second Affiliated Hospital of Nanchang University, Jiangxi, China
}

\section{Key Words}

Primordial follicle pool • Hippo signaling • Ovary cultivation in vitro

\begin{abstract}
Background: The Hippo signaling pathway, a highly conserved cell signaling system, exists in most multicellular organisms and regulates cell proliferation, differentiation, and apoptosis. It has been reported that the members of Hippo signaling are expressed in mammalian ovaries, but the exact functions of this pathway in primordial follicle development remains unclear. Methods: To analyze the spatio-temporal correlation between the core component of Hippo pathway and the size of primordial follicle pool, Western blot, Real-time PCR and immunohistochemistry were used, and the expression and localization of MST1, LATS2 and YAP1 mRNA and protein were examined in $3 \mathrm{~d}, 1 \mathrm{~m}, 5 \mathrm{~m}, 16 \mathrm{~m}$ postnatal mice ovary and the culture model of mice primordial follicle in vitro. Results: Both the protein and mRNA expression of the MST1 and LATS2 were decreased significantly as mouse age increased ( $p$ $<0.05$ ), however, the mRNA expression of them increased significantly in $16 \mathrm{~m}$ compared with $5 \mathrm{~m}$ as well as the protein expression of LATS2.The expression of YAP showed the opposite trend, and the significant protein expression of PYAP was increased before $1 \mathrm{~m}$, after which no significant change was observed. Moreover, the ratio of PYAP/YAP decreased significantly. Culturing ovaries for $8 \mathrm{~d}$ in vitro resulted in the activation of primordial follicles in $3 \mathrm{~d}$ postnatal mice ovaries, and these developed into primary follicles with the expression of PCNA increasing significantly $(p<0.05)$. The mRNA and protein expression of MST and LATS decreased significantly $(p<0.05)$, and the expression of YAP increased significantly $(p<0.05$, $p<0.01)$, whereas the ratio of PYAP/YAP decreased significantly $(p<0.05)$. Conclusion: The above results reveal that the expression of the core components of Hippo pathway changed during mouse follicular development, especially before and after primordial follicle activation

C. Xiang, J. Li and L.-L. Hu contributed equally to this paper.

Y. -H. Zheng,

and L.-P. Zheng

\section{KARGER 125}

Medical Teaching Laboratory Center, Nanchang University, XueFu road 999, Nanchang 330031,Jiangxi Province (China), and Room 103, Administration Building, Nanchang

University, XueFu road 999, Nanchang 330031, Jiangxi Province, (China)

E-Mail zhengliping@ncu.edu.cn and E-Mail yuehuizheng@163.com
\end{abstract}


in vitro. The primordial follicle activation may be related to the significant decrease of the ratio of pYAP1/YAP1. In conclusion, Hippo signaling pathway expressed in mice ovaries and have spatio-temporal correlation with the size of primordial follicle pool.

Copyright (c) 2015 S. Karger AG, Basel

\section{Introduction}

During a limited female mammalian reproductive cycle, primordial follicle stem from the primordial follicle pool, which develop though primary follicle and secondary follicle and finally become a mature follicle ovulation. However, many factors regulate the stillness of the primordial follicle pool and the primordial follicle last batch activated [1]. Historically, whether oogenesis continues after birth in mammalian ovaries remains unknown [2]. Since the 1950s [3], it has been generally accepted that females of most mammalian species lose the ability to produce oocytes at birth. At birth, germ cells in mammalian ovaries have progressed to the diplotene stage of meiotic prophase and have formed primordial follicles with surrounding somatic cells. These primordial follicles represent follicle reserves of reproductive life [4]. In recent years, a contemporary understanding of the initiation of primordial follicle growth has shown in vivo development of two classes of primordial follicles [5], the first wave of simultaneously activated follicles after birth. These primordial follicles are gradually activated in adulthood. Wu and others confirmed female germline stem cells in the ovarian surface epithelium layer that gradually activate in adulthood, as well as the formation of primordial follicles [6, 7]. In fact, only less than $1 \%$ of the follicles go through ovulation, with $99 \%$ of follicles undergoing atresia apoptosis. Therefore, clarifying the molecular mechanisms by which the initiation of primordial follicles is regulated is crucial for the prevention and treatment of female infertility and to understand premature ovarian failure.

The Hippo signaling pathway has been proven to play important roles in the regulation of stem cell proliferation, differentiation, migration and maturation, as well as establishment of normal oocyte polarity and egg chamber structure [8-11]. The core components of the Hippo pathway in mammals includes mammalian Sterile 20-like protein kinase I (MST1) and MST2 and their regulatory protein WW45 (also known as SAV1), as well as large tumor suppressor homologues 1(LATS1) and LATS2, and YAP1 (Yes-associated protein) $[12,13]$. During Drosophila oogenesis, Hippo signaling activity is required for oocyte polarization and regulation of somatic follicle-cell proliferation and differentiation in Drosophila follicle cells $[14,15]$. In mammals, overexpression of YAP enhances tumor formation and growth, whereas down-regulation of YAP decreases the tumor formation and growth in kinds of cancer cell lines [16]. The transcriptional co-activator with PDZ-binding motif (TAZ) is a transcriptional effector of the Hippo signaling cascade in regulating cell proliferation tumorigenesis [13, 17]. In breast cancer stem cells, TAZ forms a complex with the cell-polarity determinant Scribble, and loss of Scribble disrupts the activity of the core Hippo kinases MST and LATS [18]. In addition, previous results suggest that the association of YAP/TEAD (TEA domain family member) with ovarian cancer patient survival has been demonstrated, and YAP plays a role in promoting ovarian cancer cell growth in flies and humans [19-21]. On the other hand, the Hippo signaling pathway, which consists of several oncogenes (MST1/LATS2/ YAP1)could sense the changes of cell density in Drosophila ovaries [22]. The Hippo signaling pathway forms a cross network with a variety of factors and multiple signaling pathways such as Notch, TGF $\beta$, cTGF, IGF, c-erbB2, c-myc, and PI3K/AKt in different levels [23-27]. Our earlier reports demonstrated that the physiological effect of Pten and Tsc1/mTorc1 signaling pathways, which have been proved to maintain of the quiescence of primordial follicles, included anti-oncogenes that activated in the course of tumorigenesis [28-31]. However, under physiological conditions and in the in vivo cultured system throughout the entire female reproductive life span, the role of the Hippo signaling pathway in primordial follicle formation and activation has not been thoroughly investigated. 
To further verify the function and regulative mechanism of ovary, we explored the expression changes of the core components of the Hippo pathway (MST1, LATS2, YAP1) before and after primordial follicle activation and its spatio-temporal correlation with the size of primordial follicle pool in mice.

\section{Materials and Methods}

\section{Animals}

KM mice were obtained from the Center of Experimental Animals, Nan Chang University, on a 12-h light: dark cycle and were given access to food (Keao-Xieli, Beijing, China) and water ad libitum. All procedures (including animals in this study) were reviewed and approved by the ethical committee of Nan Chang University.

Ovary culture and morphometric analysis

Ovary cultivation in vitro was performed using Waymonth system (Waymonth MB 752/1 culture (Sigma, USA) supplemented with $0.23 \mathrm{mM}$ sodium pyruvate, $3 \mathrm{mg} / \mathrm{mL}$ BSA, $100 \mathrm{IU} / \mathrm{mL}$ penicillin G, $100 \mathrm{IU} / \mathrm{mL}$ streptomycin, and $10 \%$ (v/v) FBS) [27]. Briefly, under aseptic conditions, ovaries removed from neonatal female mice (3-d old), and tissues were washed with culture medium containing no serum. Then, ovaries were placed on a gelatin sponge with $600 \mu \mathrm{L}$ of culture medium in a 24-well plate, where every well contained five to six ovaries. The medium was changed every $48 \mathrm{~h}$ with replacement of half fresh medium. Experiments were repeated at least three times, with at least 20 ovaries in each experiment.

Cultured ovaries were fixed in 4\% paraformaldehyde and stained with hematoxylin and eosin. Tocompare follicle formation between in vivo and cultured ovaries, ovaries were isolated from littermate animals the same day that cultured ovaries were fixed. Follicle populations among isolated, and cultured ovaries were quantified.

Immunohistochemistry (IHC) and immunofluorescence

Immunostaining of different stage normal mice ovaries was performed according to previously published procedures (Song et al., 2002). The primary antibodies used in this study are as follows: YAP1 (1:100, ab56701), S127 phosphorylated-YAP1 (1:100, ab76252), LATS2 (1:100, ab54073), MST1 (1:100, ab51134). The secondary antibodies used in this study are Alexa 488- and Alexa 596-conjugated goat antimouse, rabbit antibodies (1:200, Molecular Probes). All of the images were taken using a NIKON Eclipse 80i microscope.

\section{Quantitative real-time PCR}

Total RNA was extracted from the different stage mice ovaries using Tri-reagent (TaKaRa). Total RNA ( $1 \mu \mathrm{g}$ in a $25-\mu \mathrm{L}$ reaction) was reversed-transcribed using PrimeScript RT reagent Kit with gDNA Eraser (TaKaRa). Amplification was performed on an ABI7000 PCR instrument (Applied Biosystems, Foster City, CA, USA) using three-stage program parameters provided by the manufacturer, as follows: 2 min at $50^{\circ} \mathrm{C}$, $2 \mathrm{~min}$ at $95^{\circ} \mathrm{C}$, and then 40 cycles of $15 \mathrm{~s}$ at $95^{\circ} \mathrm{C}$ and $30 \mathrm{~s}$ at $60^{\circ} \mathrm{C}$ and finally a cooling step at $4{ }^{\circ} \mathrm{C}$. Primers designed for amplifying list in Table 1 . The reaction was performed in $20 \mu \mathrm{L}$ reaction volume containing $0.6 \mu \mathrm{l}$ cDNA, $0.8 \mu \mathrm{L} 50 \mathrm{mM} \mathrm{MgCl}, 10 \mu \mathrm{L}$ of SYBR green master mix, $1.6 \mu \mathrm{L}$ of primers $(10 \mu \mathrm{mM})$, and $7.0 \mu \mathrm{L}$

Table 1. Primer information of main components of Hippo pathway

\begin{tabular}{|c|c|c|c|}
\hline Gene & Sequence (5'to3') & Accession & Amplified fragment length (bp) \\
\hline \multirow[t]{2}{*}{ MST1 } & F:CAGCCCGAGGAAGTGTTTGA & XM_006499963.1 & $171-287$ \\
\hline & R:CACGGGCACTTGCTTGATTG & & \\
\hline \multirow[t]{2}{*}{ LATS2 } & F:CTTTGCTTCCCTCGGACAGT & NM_010690.1 & $6213-6358$ \\
\hline & R:AACTGGGTGTAGTCAGGGGT & & \\
\hline \multirow[t]{2}{*}{ YAP1 } & F:TCCAACCAGCAGCAGCAAAT & NM_001171147 & $1060-1157$ \\
\hline & R:TTCCGTATTGCCTGCCTGCCGAAA & & \\
\hline \multirow[t]{2}{*}{$\beta$-actin } & F:ACGTGCCGCCTGGAGAAAC & XM_001476707.3 & $785-886$ \\
\hline & R:GTCCTCAGTGTAGCCCAAGATGC & & \\
\hline
\end{tabular}


of nuclease-free water per sample. Every sample was amplified in triplicate to normalize the system and pipetting error, using the standard curve method with $\beta$-actin as the reference gene.

\section{Western blot analysis}

Total protein was extracted from ovarian tissue with RIPA lysis solution (Beyotime, P0013C) for 30 min on ice with frequent vortexing, and then the lysates were collected by centrifugation at $1000 \mathrm{rpm}$ for $5 \mathrm{~min}$ at $4^{\circ} \mathrm{C}$ and combined the sample loading buffer for $4: 1$. The proteins were subjected to SDS-PAGE (sodium dodecyl sulfate-polyacrylamide gel electrophoresis) transferred to PVDF membranes (Millipore Corp., Bedford, MA) and processed per antibody manufacturer's instruction. The primary antibodies were: YAP1 (1:100, ab56701, Abcam), S127 phosphorylated-YAP1 (1:10000, ab76252, Abcam), MST1 (1:10000, ab51134, Abcam), LATS2 (1:100, ab54073, Abcam), $\beta$-actin (1:1000, ab1801, Abcam) and PCNA (1:1000, ab18197, Abcam). The band intensity was quantified using $\beta$-actin as internal quantitative control. Each experiment was duplicated at least three times.

\section{Statistical methods}

For each set of data, all experiments were conducted at least in triplicate and repeated at least three times, representing the mean \pm SEM, within an individual experiment. The statistical comparisons among different age groups were analyzed by Student's paired t test. The threshold $\mathrm{p}<0.05$ was considered significant, $p<0.01$ and $p<0.001$ were considered extremely significant. All analyses were performed using GraphPad Prism 5.0 (GraphPad Software, Inc., San Diego, CA).

\section{Results}

Components of Hippo signaling are expressed in different stages in mice ovaries

We chose $3 \mathrm{~d}, 1 \mathrm{~m}, 5 \mathrm{~m}$ and $16 \mathrm{~m}$ of mice ovaries, respectively. Western blot and RT-PCR assays were used to detect MST1, LATS2, YAP1, and pYAP1 expression. The western blot results showed that the expression of MST1 and LATS2 proteins were the highest in $3 \mathrm{~d}$ mice ovaries, and the MST1 protein had no significant difference among the $1 \mathrm{~m}, 5 \mathrm{~m}, 16 \mathrm{~m}$ groups $(\mathrm{p}>0.05)$. With the increase of age, MST1 content significantly down-regulated $(\mathrm{p}<$ 0.05), and LATS2 protein expression change trend of the MST1 ( $<<0.05, p<0.01, p<0.05)$, but the LATS2 protein of $16 \mathrm{~m}$ significantly increased compared with $5 \mathrm{~m}(\mathrm{p}<0.05)$ (Fig. 1A, B). However, YAP1 protein expressed was lowest in the $3 \mathrm{~d}$ group because for the $1 \mathrm{~m}, 5 \mathrm{~m}$, 16 m groups, expression increased significantly ( $p<0.05, p<0.01, p<0.01)$, and reduced significantly between the $16 \mathrm{~m}$ and $5 \mathrm{~m}$ groups $(\mathrm{p}<0.05)$. Similar to the YAP1 protein, the p-YAP1 protein also displayed the lowest expression in the $3 \mathrm{~d}$ group ( $<$ < 0.05) (Fig. 1C, D), but these three groups had no significant difference. With the increase in age of mice, the value of pYAP1/YAP1 decreased ( $\mathrm{p}<0.05)$.

Subsequently, we performed real-time PCR experiments to determine whether Hippo family genes MST1 and LATS2 mRNA strongly expressed by $3 \mathrm{~d}$ mice ovaries and downregulation persisted from $1 \mathrm{~m}, 5 \mathrm{~m}$ to $16 \mathrm{~m}(\mathrm{p}<0.05, \mathrm{p}<0.01, \mathrm{p}<0.01)$, but the level of $16 \mathrm{~m}$ was higher than $5 \mathrm{~m}$ (Fig. 1E). Conversely, the relative mRNA expression of YAP1 was significantly lower than the $3 \mathrm{~d}$ mice ovaries compared with the other three groups $(\mathrm{p}<0.01$, $\mathrm{p}<0.001, \mathrm{p}<0.01$ ), with the level of $16 \mathrm{~m}$ significantly decreased compared with the level of $5 \mathrm{~m}(\mathrm{p}<0.05)$. Specifically, the relative MST, LATS, and YAP expression of micrographs were similar to the molecular results.

\section{Expression of components of the Hippo signaling pathway in follicle development}

The micrographs of ovaries in $3 \mathrm{~d}, 1 \mathrm{~m}, 5 \mathrm{~m}$ and $16 \mathrm{~m}$ mice were used to determine the cellular localization of MST1, LATS2, YAP1, and pYAP1 genes (Fig. 2a). The results showed the expression of MST1, LATS2, and YAP1 mainly in the oocyte cytoplasm, but light MST1, LATS2, and YAP1 staining were also observed in the cytoplasm, perivitelline membranes, granulose cells of primary follicles, secondary follicles, and mature follicles. Whereas the expression of MST1, LATS2, and YAP1 in secondary follicles was at higher levels than granulose cells, there 
A

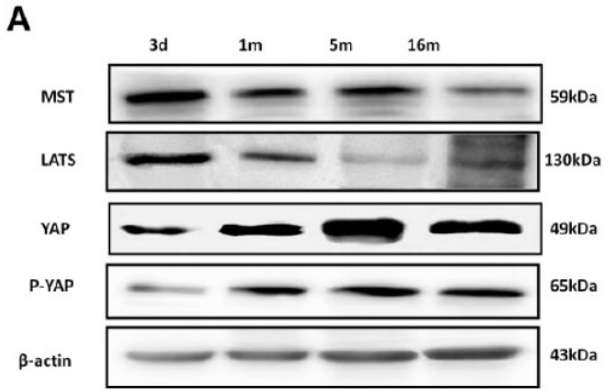

C

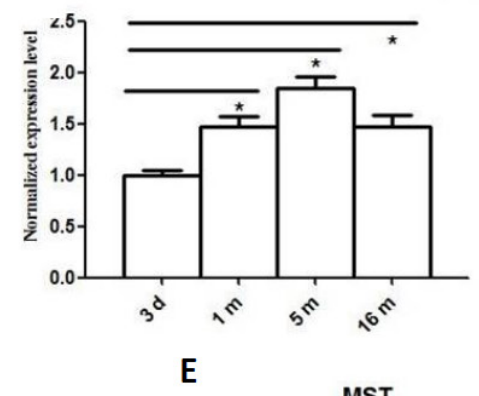

\section{B}

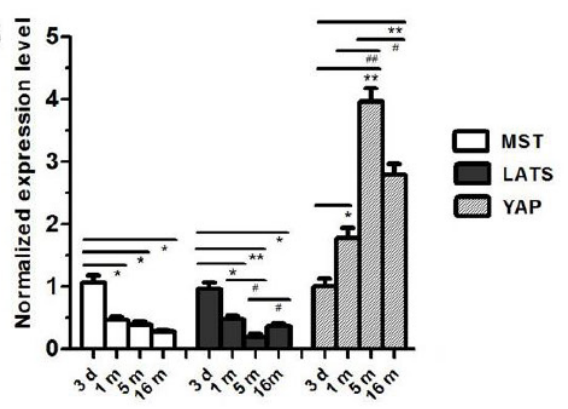

D

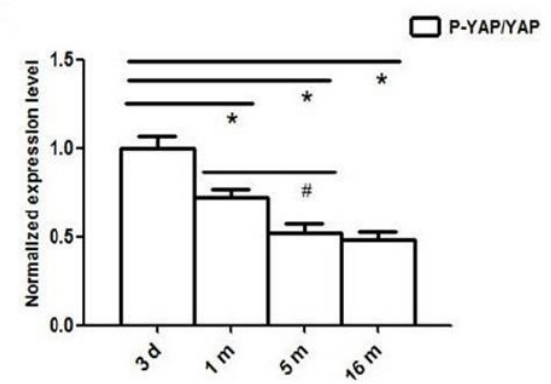

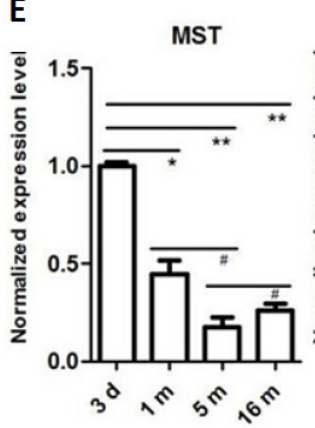
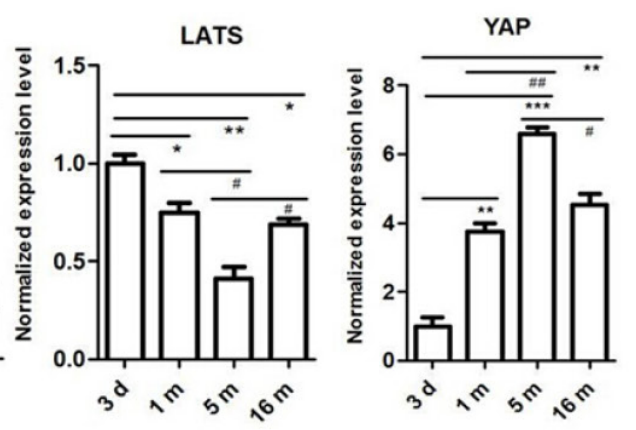

F MST1
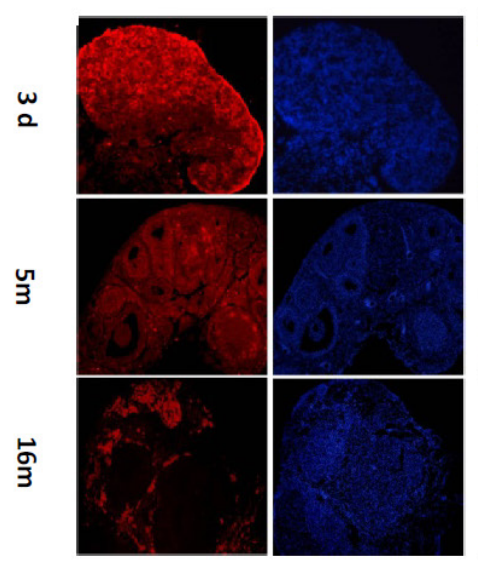

LATS2
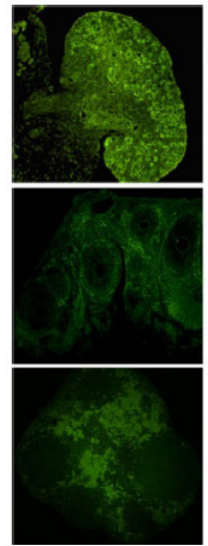

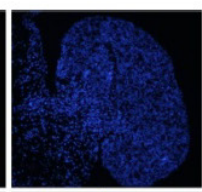

YAP1
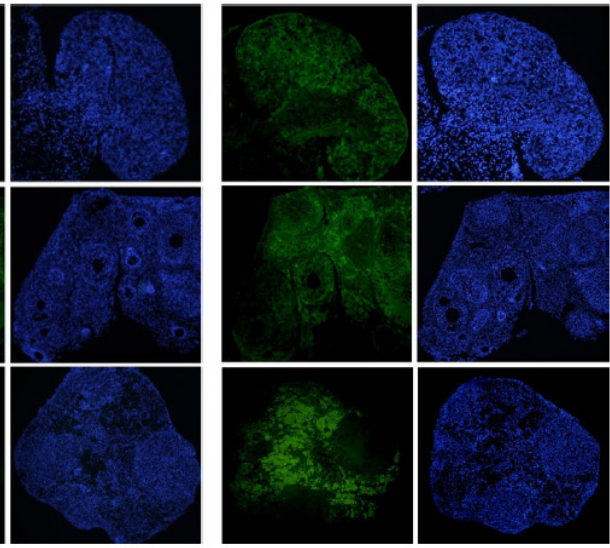

Fig. 1. Expression of Hippo families genes in different stage of mice ovaries. A: Western blot analysis for MST1, LATS2, YAP1, and pYAP1 expressing in $3 \mathrm{~d}, 1 \mathrm{~m}, 5 \mathrm{~m}$ and $16 \mathrm{~m}$ of mice ovaries. B: Quantification of western blot analysis for MST1, LATS2, and YAP1. C: Quantification of western blot analysis for pYAP1. D: The value of pYAP1/YAP1. E: Quantitative RT-PCR analyses of expression of Hippo member for $3 \mathrm{~d}, 1 \mathrm{~m}, 5$ $\mathrm{m}$, and $16 \mathrm{~m}$ mice ovaries. The results present as the mean $\pm \mathrm{SD} .{ }^{*} \mathrm{p}<0.05,{ }^{* *} \mathrm{p}<0.01$, compared with the $3 \mathrm{~d}$ group; \# $\mathrm{p}<0.05$, \#\# $\mathrm{p}<0.01$, compared with the adjacent group. F: Micrographs depict MST1, LATS2, and YAP1 during $3 \mathrm{~d}, 5 \mathrm{~m}$, and $16 \mathrm{~m}$ of mice ovaries as detected by anti-MST1 antibody (red), anti-LATS2 antibody (green), and anti-YAP1 antibody (green). The nucleus was stained by DAPI (blue). 
a
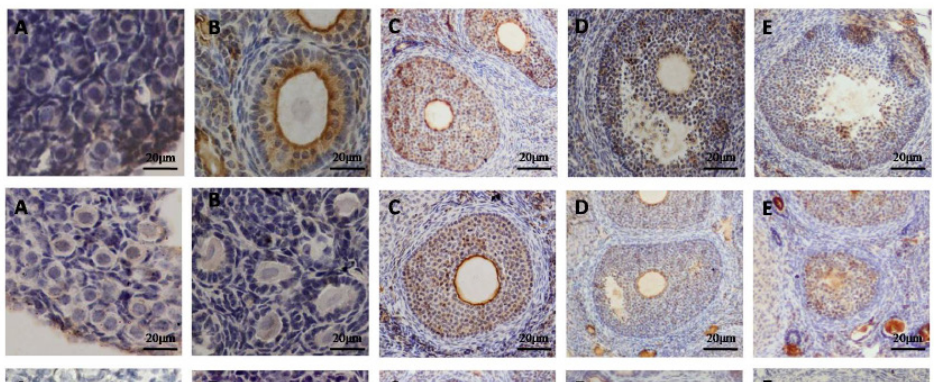

$\underset{\mathfrak{1}}{3}$
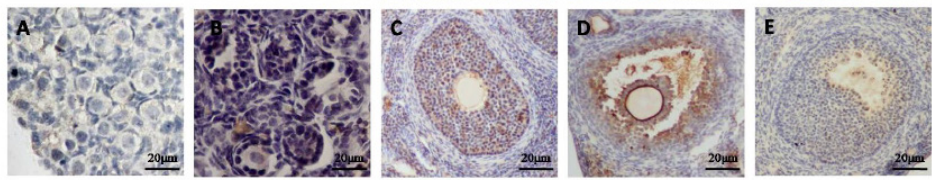

$\frac{5}{\sqrt{n}}$
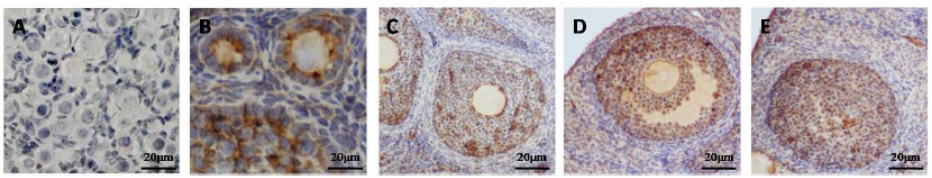

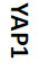

b

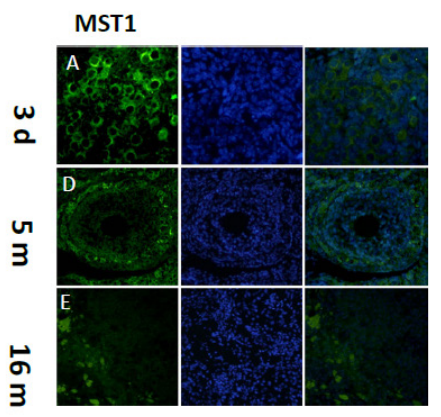

LATS2
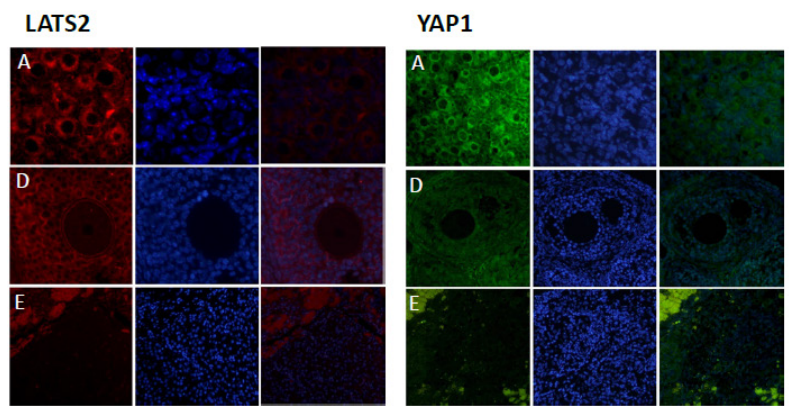

Fig. 2. Distribution of Hippo families genes in different stage of mice ovarian follicle. Histological sections of the $3 \mathrm{~d}, 5 \mathrm{~m}$ and $16 \mathrm{~m}$ of mice ovaries. Experiments were repeated three times and representative images are shown. The scale bars $20 \mu \mathrm{m}$. a: Immunocytochemical localization of Hippo components in different stage of follicles by anti-MST1 antibody, anti-LATS2 antibody and anti-YAP1 antibody. b: Micrographs depict MST1, LATS2, and YAP1 during $3 \mathrm{~d}, 5 \mathrm{~m}$ and $16 \mathrm{~m}$ of mice ovaries as detected by anti-MST1 antibody (red), antiLATS2 antibody (green) anti-YAP1 antibody (green). The nucleus was stained by DAPI (blue). (A: Primordial follicles; B: Primary follicles; C: Secondly follicles; D: Mature follicles; E: Atresia follicles).

was less or no staining in atresia follicles. However, pYAP1 was also localized in oocytes of primordial follicles and granulose cells, oocytes, perivitelline membranes of primary follicles, secondary follicles and mature follicles but at low levels in the granulosa cells of the atretic follicle. The study reveals that the Hippo family's genes are expressed in early granulose cells and shows that the Hippo signaling pathway is active at the time of follicle assembly.

The establishment of in vitro ovary culture and identification

Collectively, our gene expression and immunohistochemistry data support the hypothesis that Hippo signaling occurs during early folliculogenesis, but how Hippo functions during this particular period of ovary development is unknown. In this system, neonatal mice ovaries are maintained in culture for $8 \mathrm{~d}$, a time span that allows for follicle formation. The HE staining results (Fig. 3a, b) showed that the complete cultured ovarian structure and its corresponding volume is increased, and it contained primary follicles in the medulla of the ovary and primordial follicles in the ovarian cortex. The western blotting and RT-PCR experiments verified that PCNA gene [32] of cultured ovaries is up-regulated compared with 
$\mathbf{a}$

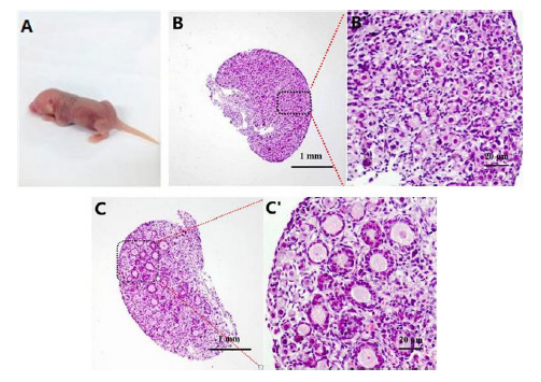

c

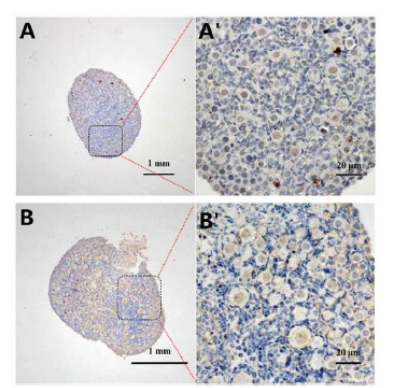

b

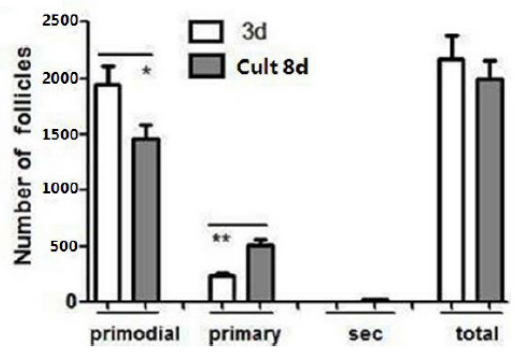

e

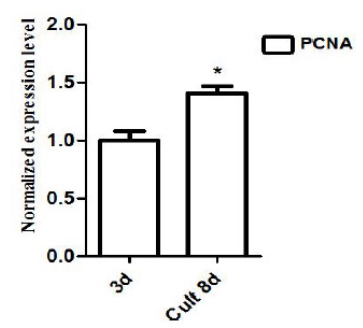

Fig. 3. The changes of primordial follicles between $3 \mathrm{~d}$ mice ovaries and ovaries cultured $8 \mathrm{~d}$ in vitro. $\mathrm{a}, \mathrm{b}$ : Quantity changes of primordial follicles after culturing $8 \mathrm{~d}$ in vitro; $\mathrm{c}$ : A-A' pictures show the expression of PCNA in $3 \mathrm{~d}$ mice ovaries by immunohistochemistry; B-B' pictures show the expression of PCNA in cultured mice ovaries for $3 \mathrm{~d}$ and cult $8 \mathrm{~d}$ by immunohistochemistry; d-e: Protein expression of PCNA after cultured mice ovaries for $8 \mathrm{~d} ; * \mathrm{p}<0.05$.

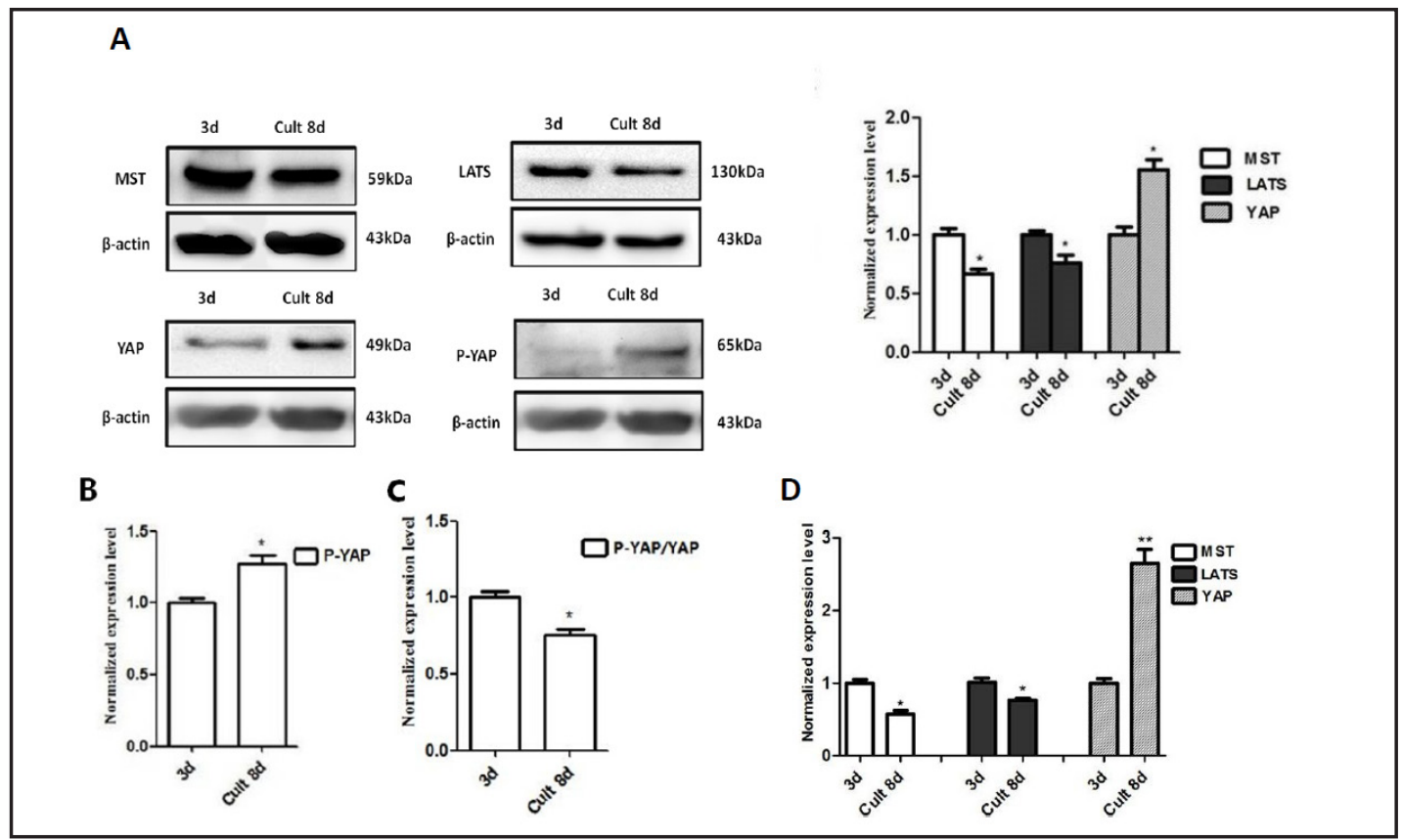

Fig. 4. Expression changes of MST1, LATS2, and YAP1 in primordial follicles after cultured $8 \mathrm{~d}$ in vitro. A: Western blot analysis(left) for MST1, LATS2, YAP1, pYAP1 and $\beta$-actin expressing in $3 \mathrm{~d}$ and cultured $8 \mathrm{~d}$ in vitro of mice ovaries, and quantification of western blot analysis for MST1, LATS2, and YAP1 (right). B: Quantification of western blot analysis for pYAP1. C: The value of pYAP1/YAP1. D: Quantitative RT-PCR analyses of expression of Hippo family genes between $3 \mathrm{~d}$ mice ovaries and cultured $8 \mathrm{~d}$ in vitro are shown. The results present as the mean $\pm \mathrm{SD}$. ${ }^{*} p<0.05,{ }^{* *} p<0.01$, compared with the 3 d group; $\# p<0.05$, \#\# $p<0.01$, compared with the adjacent group. 
ovaries isolated from $3 \mathrm{~d}$ mice $(\mathrm{p}<0.05)$. These results suggest that the development of mice ovaries is well, and most of the primordial follicles have been formatted and activated. We have found that expression of Hippo genes in different stage mice ovaries were downregulated using immunostaining of tissue sections and molecular methods. In this study, to examine how Hippo signal pathway involves in the formation of primordial follicles, we compared ovaries cultured for $8 \mathrm{~d}$ and in vivo with ovaries cultured for $3 \mathrm{~d}$ using western blotting and real-time PCR. The relative mRNA and total protein of expression of MST1, LATS2 were significantly lower in cultured ovaries than in vivo ovaries $(\mathrm{p}<0.05)$ (Fig. 4A, D). However, YAP1 was increase in cultured ovaries ( $<0.05, p<0.01)$. Moreover, protein expression of pYAP1 displayed extremely increased in cultured ovaries ( $p<0.05$, but the value of $\mathrm{pYAP1}$ /YAP1 show a significant decrease $(\mathrm{p}<0.05)$ (Fig. 4B, C). These results suggest that Hippo signaling pathway affects primordial follicle formation.

\section{Discussion}

Ovary morphometric analysis show that the number of primordial follicles is highest in $3 \mathrm{~d}$ mice ovaries ( $80 \%$ in total follicles), and a large number of growing follicles at different levels in $1 \mathrm{~m}$ mice ovaries. When mice ovaries are developed to $5 \mathrm{~m}$, more follicle growth and corpus luteum formation occurs, whereas in $16 \mathrm{~m}$ mice, there are no normal follicles except atretic follicles [33]. Therefore, we chose $3 \mathrm{~d}, 1 \mathrm{~m}, 5 \mathrm{~m}$ and $16 \mathrm{~m}$ mice ovaries as experimental groups in this study to reflect the size of the primordial follicle pool in the reproductive life of female mice.

As one of the most crucial cell signaling pathways, the Hippo signaling pathway regulates female reproductive system development [34-38]. We provide evidence for the expression of Hippo pathway genes in the different stage mouse ovaries and propose a novel role for Hippo signaling in regulating primordial follicle formation. LATS2 and MST1 are maximally expressed in the neonatal mouse ovary ( 3 d group) compared with the protein level of YAP1. However, as the age of mice develop, the primordial follicles start developing unceasingly. While follicular growth is accompanied by oocytes maturation and granular cell proliferation, MST1 and LATS2 are down-regulated during follicle formation with age, and the expression level of YAP1 is up-regulated all of the time. YAP1, a transcription factor, is an ovarian cancer oncogene in the previous studies, but the core of the mammalian Hippo pathway is composed of two kinases complexes: the MST1-2/Sav1 complex and the LATS2/MOB complex [21, 39, 40]. The previous study showed that YAP1 has no transcriptional activity and its actions are dependent on downstream transcriptional factors [41, 42]. As shown in Fig. 1C, decreases in pYAP1 levels occur along with as well as a decrease in the value of pYAP1/YAP1. Meanwhile, MST1, LATS2, and YAP1 specifically express in the cytoplasm of oocytes (Fig. 2b); however, for MST1, LATS2, and YAP1 not expressed in the cytoplasm, the different expression and localization may suggest MST1, LATS2, and YAP1 provide a potential role for transition from primordial to primary follicles. pYAP1 begins expression in oocytes but later weakly expresses in granulosa cells of atresia follicles. Therefore, we conclude that the dynamic change in the Hippo signaling pathway corresponds with the follicle development schedule.

Interestingly, MST1 mRNA levels are not consistent with the protein expression level in the $16 \mathrm{~m}$ age. The amount of MST1 protein expression shows no significant difference within the different stages of development in mice ovaries, but the amount of MST1 mRNA expression is significantly increased. However, we suggest these reasons for this unique phenomenon: First, RNA is converted into protein by a complex regulatory process [43-45]. The MST1 gene in ovarian tissue in aging mice is transcribed into mRNA after degradation, or disturbed by other signals leading to a suspension of translation, resulting in the MST1 protein level having no significant difference. Second, the eukaryotic expression system has a modification process known as post-translational processing [46]. If protein processing after translation is not correct, MST1 protein may be degraded with endopeptidase, and activity may also be decreased, or lead to lack the required core components for translation

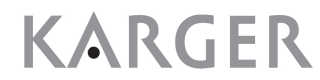


into protein in the ovarian tissue of ageing mice. This will finally cause the change of protein levels. Third, there may be some type of regulatory mechanism to restrain MST1 protein expression in the ovarian tissue of ageing mice and in the MST1 mRNA level there may be feedback suppression allowing it to express.

It is becoming clear that the key components of the Hippo signaling pathway show differential expression pattern under different mouse life stages because of follicle development, suggesting that MST1, LATS2, and YAP1 are in part coordinated through oocyte maturation, granular cell proliferation, follicular atresia, and other physiological processes. Consistent with the role of Hippo signaling genes in regulating follicle growth [34], ovaries were removed from infertile patients, followed by fragmentation to disrupt Hippo signaling. After grafting ovarian tissues back to patients, rapid follicle growth were found in some patients. Likewise, damage incurred by cutting or drilling PCOS ovaries could enhance actin polymerization and disrupt Hippo signaling to promote follicle growth [34, 47, 48]. Although Chen et al. confirmed that YAP1 appears to be a new susceptibility gene for PCOS in Han Chinese women [49], we cannot exclude more localized changes in Hippo genes expression at the cellular level. Furthermore, LATS1 phosphorylated forkhead L2 (FOXL2) and regulated its transcriptional activity in some ovary of primary ovarian insufficient POI patients [50, 51]. In this study, our results also confirmed that the Hippo signal pathway relates with the maturation of mammalian oocytes, proliferation of granulosa cells and follicular atresia in folliculogenesis.

The previous study reported rodent ovary culture has been successfully performed by other investigators to confirm roles for signaling molecules during early follicle formation $[24,27,52]$. We utilize an in vivo follicle formation model in this system, arguing that factors important for follicle assembly are important to ovarian development. Neonatal mouse ovaries maintained in culture were treated by in vivo culture system to address functional roles for Hippo in the neonatal mouse ovary (Fig. 3a). We used PCNA to assess the Hippo signaling pathway because PCNA was originally identified as an antigen that is expressed in the nuclei of cells during the DNA synthesis phase of the cell cycle, and it plays an important role in cell proliferation to start and is a good indicator of the state of cell proliferation (Fig. $3 c$ ). The study also verified this culture model is useful for investigation of folliculogenesis mechanisms.

In our study, YAP1 and pYAP1 total proteins are increased, respectively, but the level expression of pYAP1 shows a decrease in the value of pYAP1/YAP1. This finding indicates that the level expression of pYAP1 is up-regulated after primordial follicular activation. In a previous study, pYAP1, a transcription factor, can enter into the nucleus and play a potential role as in mediating follicular proliferation. Whereas high cell-density activates the Hippo pathway kinases and promotes the phosphorylation of YAP1 on serine 127, this leads to its nuclear export and growth inhibition [22]. Therefore, we can conclude that the Hippo signaling pathway affects primordial follicle formation by increasing expression of pYAP1 in oocytes nuclei.

However, this cornerstone of mammalian female reproductive biology has been recently challenged by a growing body of evidence showing the isolation and propagation of germ stem cells from mouse and human ovaries [53]. Some experimental evidence has shown that the first wave of follicles exists in the cytoplasm of ovaries for $3 \mathrm{~m}$ and contributes to the onset of puberty and to early fertility. However, primordial follicles at the ovarian cortex are formed after birth and gradually replace the first wave of follicles until they become the sole source of follicles from $3 \mathrm{~m}$ until the end of the reproductive life. Our data clearly show that expression of MST1 and LATS2 at ovarian cortex were significantly higher than ovarian medulla. Recently, some previous studies have also confirmed that similar to Hippo signaling pathway, Tsc/mTORC1 signaling and PTEN/PI3K (phosphatidylinositol 3 kinase) signaling synergistically regulate the dormancy and activation of primordial follicles, and together ensure the proper length of female reproductive life [54, 55]. Furthermore, our previous research also confirmed that $c$-src was expressed in mammalian ovaries, which plays an important role in primordial follicle activation and development [25]. Similarly, YAP1 is an 
evolutionarily conserved proto-oncogene that shows a similar expression before and after progressive activation of primordial follicles. Therefore, we include that the MST1-LATS2YAP1-Hippo signaling pathway may decrease primordial follicles activation and formation. However, the above results are only based on core components of Hippo pathway (MST1, LATS2, and YAP1) molecular expression and morphological basis.

This study presents some novel evidence that clarifies the Hippo signaling pathway regulates primordial follicles formation and activation under physiological conditions and in vivo cultured system, which provides a solid foundation for studying how the majority of ovarian primordial follicles are maintained in a quiescent state to provide a reserve for continuous reproductive success.

\section{Acknowledgments}

We are thankful to Life Sciences Institute of Nanchang University for providing a high level of scientific research conditions. We thank all of the members in our lab. The study was supported by the National Natural Science Foundation of China (No. 81260098, No. 81360100) and the Excellence 555 Engineering of Jiangxi province.

\section{References}

1 Lawrence PA, Casal J: The mechanisms of planar cell polarity, growth and the Hippo pathway: Some known unknowns. Dev Biol 2013;377:1-8.

2 Kezele P, Nilsson E, Skinner MK: Cell-cell interactions in primordial follicle assembly and development. Front Bio 2002;7:1990-1996.

3 Mandl AM, Zuckerman S: The growth of the oocyte and follicle in the adult rat. J Endocrinol 1952;8:126132.

4 Reddy P, Zheng W, Liu K: Mechanisms maintaining the dormancy and survival of mammalian primordial follicles. Trends Endocrin Met 2010;21:96-103.

5 Zheng W, Zhang H, Gorre N, Risal S, Shen Y, Liu K: Two classes of ovarian primordial follicles exhibit distinct developmental dynamics and physiological functions. Hum Mol Genet 2014;23:920-928.

6 Zou K, Yuan Z, Yang Z, Luo H, Sun K, Zhou L, Xiang J, Shi L, Yu Q, Zhang Y, Hou R, Wu J: Production of offspring from a germline stem cell line derived from neonatal ovaries. Nat Cell Biol 2009;11:631-636.

7 Shi L, Wu J: Epigenetic regulation in mammalian preimplantation embryo development. Reprod Biol Endocrin 2009; 7:59.

-8 Polesello C, Tapon N: Salvador-warts-hippo signaling promotes Drosophila posterior follicle cell maturation downstream of notch. Curr Biol 2007;17:1864-1870.

9 Barry ER, Camargo FD: The Hippo superhighway: signaling crossroads converging on the Hippo/Yap pathway in stem cells and development. Curr Opin Cell Biol 2013;25:247-253.

-10 Karpowicz P, Perez J, Perrimon N: The Hippo tumor suppressor pathway regulates intestinal stem cell regeneration. Development 2010;137:4135-4145.

11 Hao J, Zhang Y, Jing D, Li Y, Li J, Zhao Z: Role of Hippo signaling in cancer stem cells. J Cell Physiol 2014;229:266-270.

12 Zhao B, Lei QY, Guan KL: The Hippo-YAP pathway: new connections between regulation of organ size and cancer. Curr Opin Cell Biol 2008;20:638-646.

13 Lei QY, Zhang H, Zhao B, Zha ZY, Bai F, Pei XH, Zhao S, Xiong Y, Guan KL: TAZ promotes cell proliferation and epithelial-mesenchymal transition and is inhibited by the hippo pathway. Mol Cell Biol 2008;28:24262436.

14 Yan Y, Denef N, Tang C, Schupbach T: Drosophila PI4KIIIalpha is required in follicle cells for oocyte polarization and Hippo signaling. Development 2011;138:1697-1703.

15 Klusza S, Deng WM: At the crossroads of differentiation and proliferation: Precise control of cell-cycle changes by multiple signaling pathways in Drosophila follicle cells. Bioessays 2011;33:124-134. 


\section{Cellular Physiology Cell Physiol Biochem 2015;35:957-968 and Biochemistry

-16 Fu D, Lv XM, Hua GH, He CB, Dong JX, Lele SM, Li DWC, Zhai QL, D JS, Wang C: YAP Regulates Cell Proliferation, Migration, and Steroidogenesis in Adult Granulosa Cell Tumors. Endocr Relat Cancer 2014;21:297-310.

17 Pan D: The hippo signaling pathway in development and cancer. Dev Cell 2010;19:491-505.

- 18 Cordenonsi M, Zanconato F, Azzolin L, Forcato M, Rosato A, Frasson C, Inui M, Montagner M, Parenti AR, Poletti A, Daidone MG, Dupont S, Basso G, Bicciato S, Piccolo S: The Hippo transducer TAZ confers cancer stem cell-related traits on breast cancer cells. Cell 2011;147:759-772.

19 Xia Y, Chang T, Wang Y, Liu Y, Li W, Li M, Fan HY: YAP promotes ovarian cancer cell tumorigenesis and is indicative of a poor prognosis for ovarian cancer patients. PLoS One 2014;9:e91770.

20 Hall CA, Wang R, Miao J, Oliva E, Shen X, Wheeler T, Hilsenbeck SG, Orsulic S, Goode S: Hippo pathway effector Yap is an ovarian cancer oncogene. Cancer Res 2010;70:8517-8525.

21 Zhang X, George J, Deb S, Degoutin JL, Takano EA, Fox SB, AOCS Study group, Bowtell DD, Harvey KF: The Hippo pathway transcriptional co-activator, YAP, is an ovarian cancer oncogene. Oncogene 2011;30:28102822.

-22 Zhang J, Smolen GA, Haber DA: Negative regulation of YAP by LATS1 underscores evolutionary conservation of the Drosophila Hippo pathway. Cancer Res 2008;68:2789-2794.

23 Chen HJ, Wang CM, Wang TW, Liaw GJ, Hsu TH, Lin TH, Yu JY: The Hippo pathway controls polar cell fate through Notch signaling during Drosophila oogenesis. Dev Biol 2011;357:370-379.

24 Chen CL, Fu XF, Wang LQ, Wang JJ, Ma HG, Cheng SF, Hou ZM, Ma JM, Quan GB, Shen W, Li L: Primordial follicle assembly was regulated by Notch signaling pathway in the mice. Mol Biol Rep 2014;41:1891-1899.

25 Du XY, Huang J, Xu LQ, Tang DF, Wu L, Zhang LX, Pan XL, Chen WY, Zheng LP, Zheng YH: The proto-oncogene c-src is involved in primordial follicle activation through the PI3K, PKC and MAPK signaling pathways. Reprod Biol Endocrin 2012;10:58.

-26 Neto-Silva RM, de Beco S, Johnston LA: Evidence for a Growth-Stabilizing Regulatory Feedback Mechanism between Myc and Yorkie, the Drosophila Homolog of Yap. Dev Cell 2010;19:507-520.

27 Zheng LP, Zhang DL, Huang J, Xu LQ, Xu AX, Du XY, Tang DF, Zheng YH: Proto-oncogene c-erbB2 initiates rat primordial follicle growth via PKC and MAPK pathways. Reprod Biol Endocrin 2010;8:66.

28 Tong Y, Li F, Lu Y, Cao Y, Gao J, Liu J: Rapamycin-sensitive mTORC1 signaling is involved in physiological primordial follicle activation in mouse ovary. Mol Reprod Dev 2013;80:1018-1034.

29 Adhikari D, Gorre N, Risal S, Zhao Z, Zhang H, Shen Y, Liu K: The safe use of a PTEN inhibitor for the activation of dormant mouse primordial follicles and generation of fertilizable eggs. PLoS One 2012; 7:e39034.

-30 Deepak adhikari, Lui K: mTORC signaling in the control of activation of primordial follicles. Cell Cycle 2010;9:1673-1674.

-31 McLaughlin M, Kinnel HL, Anderson RA, Telfer EE: Inhibition of phosphatase and tensin homolog (PTEN) in human ovary in vitro results in increased activation of primordial follicles but compromises development of growing follicles. Mol Hum Reprod 2014;20:736-744.

-32 Zhang XY, Wu FY, He JH, Liao XL, Wang W, Jiang JW: Construction of shRNA Targeting PCNA Gene and Its Effects on Proliferation and Apoptosis of HepG2 Cell Lines. Progress Biochem 2010;37:278-287.

- 33 Johnson J, Canning J, Kaneko T, Pru JK, Tilly JL: Germline stem cells and follicular renewal in the postnatal mammalian ovary. Nature 2004;428:145-150.

-34 Kawamura K, Cheng Y, Suzuki N, Deguchi M, Sato Y, Takae S, Ho CH, Kawamura N, Tamura M, Hashimoto S, Sugishita Y, Morimoto Y, Hosoi Y, Yoshioka N, Ishizuka B, Hsueh AJ:Hippo signaling disruption and Akts timulation of ovarian follicles for infertility treatment. Proc Natl Acad Sci 2013;110:17474-17479.

-35 Moya IM, Halder G: Discovering the Hippo pathway protein-protein interactome. Cell Res 2014;24:137-138.

-36 Sun S, Zhao S, Wang Z: Genes of Hippo signaling network act unconventionally in the control of germline proliferation in Drosophila. Dev Dyn 2008;237:270-275.

-37 Badouel C, Garg A, McNeill H: Herding Hippos: regulating growth in flies and man. Curr Opin Cell Biol 2009;21:837-843.

38 An Y, Kang Q, Zhao Y, Hu X, Li N: Lats2 modulates adipocyte proliferation and differentiation via hippo signaling. PLoS One 2013;8:e72042.

-39 Halder G, Johnson RL: Hippo signaling: growth control and beyond. Development 2011;138:9-22.

40 Visser S, Yang X: LATS Tumor Suppressor: A new governor of cellular homeostasis. Cell Cycle 2010;9:38923903. 


\section{Cellular Physiology $\quad$ Cell Physiol Biochem 2015;35:957-968 and Biochemistry

41 Lamar JM, Stern P, Liu H, Schindler JW, Jiang ZG, Hynes RO: The Hippo pathway target, YAP, promotes metastasis through its TEAD-interaction domain. Proc Natl Acad Sci 2012;109:e2441-2450.

-42 Hau JC, Erdmann D, Mesrouze Y, Furet P, Fontana P, Zimmermann C, Schmelzle T, Hofmann F, Chène P:The TEAD4-YAP/TAZ protein-protein interaction: expected similarities and unexpected differences. Chembiochem 2013;14:1218-1225.

43 Pichon X, Wilson LA, Stoneley M, Bastide A, King HA, Somers J, Willis AE:RNA binding protein/RNA element interactions and the control of translation. Curr Protein Pept Sci 2012;13:294-304.

-44 Freischmidt A, Liss M, Wagner R, Kalbitzer HR, Horn G: RNA secondary structure and in vitro translation efficiency. Protein Expr Purif 2012;82:26-31.

45 Thach RE, Sundararajan TA, Dewey KF, Brown JC, Doty P: Translation of synthetic messenger RNA. Cold Spring Harb Symp Quant Biol 1966;31:85-97.

46 Papa S, Scacco S, De Rasmo D, Signorile A, Papa F, Panelli D, Nicastro A, Scaringi R, Santeramo A, Roca E, Trentadue R, Larizza M:cAMP-dependent protein kinase regulates post-translational processing and expression of complex I subunits in mammalian cells. Biochim Biophys Acta 2010;1797:649-658.

-47 Lunde 0, Djoseland 0, Grottum P: Polycystic ovarian syndrome: a follow-up study on fertility and menstrual pattern in 149 patients 15-25 years after ovarian wedge resection. Hum Reprod 2001;16:1479-1485.

-48 Farquhar C, Brown J, Marjoribanks J: Laparoscopic drilling by diathermy or laser for ovulation induction in anovulatory polycystic ovary syndrome. Cochrane Database Syst Rev Cochrane Database Syst Rev 2012 Jun 13;6:CD001122. doi: 10.1002/14651858.CD001122.pub4.

-49 Li T, Zhao H, Zhao XM, Zhang B, Cui L, Shi Y, Li G, Wang P, Chen ZJ: Identification of YAP1 as a novel susceptibility gene for polycystic ovary syndrome. J Med Genet 2012;49:254-257.

50 St John MA, Tao W, Fei X, Fukumoto R, Carcangiu ML, Brownstein DG, Parlow AF, McGrath J, Xu T: Mice deficient of Lats1 develop soft-tissue sarcomas, ovarian tumours and pituitary dysfunction. Nat Genet 1999;21:182-186.

-51 Pisarska MD, Kuo FT, Bentsi-Barnes IK, Khan S, Barlow GM: LATS1 phosphorylates forkhead L2 and regulates its transcriptional activity. Am J Physiol Endoc M 2010;299:e101-109.

52 Kezele P, Skinner MK: Regulation of ovarian primordial follicle assembly and development by estrogen and progesterone: endocrine model of follicle assembly. Endocrinology 2003;144:3329-3337.

53 Evron A, Blumenfeld Z: Ovarian Stem Cells-the Pros and Cons. Clin Med Insights Reprod Health 20137:4347.

-54 Adhikari D, Zheng W, Shen Y, Gorre N, Hamalainen T, Cooney AJ, Huhtaniemi I, Lan ZJ, Liu K: Tsc/mTORC1 signaling in oocytes governs the quiescence and activation of primordial follicles. Hum Mol Genet 2010;19:397-410.

-55 Li J, Kawamur K, Cheng Y, Liu S, Klein C, Liu S, Duan EK, Hsueh AJ: Activation of dormant ovarian follicles to generate mature eggs. Proc Natl Acad Sci 2010;107:10280-10284. 